\title{
Manifestação clínica de peritonite em pacientes que vivem com insuficiência renal crônica
}

\author{
Clinical signs of peritonitis in patients living with chronic renal failure
}

José Jacy Ferreira', Modesto Leite Rolim Neto², Cícero Hedilberto Pereira Filgueiras Macêdo ${ }^{3}$, Jesus de Sousa Cartaxo ${ }^{4}$, Nádia Nara Rolim Lima², Lara Eduardo de Galiza², Rose Heidy Costa Silva², Émile Bevilaqua de Carvalho Costa²

\begin{abstract}
Resumo
Introdução: A diálise peritoneal é uma alternativa segura e eficaz no tratamento de pacientes com insuficiência renal crônica terminal. Desde a introdução da diálise peritoneal, a peritonite tem sido a principal complicação dessa técnica. Objetivo: Analisar a frequência da peritonite num centro de hemodiálise, situado no sertão da Paraíba, de 2005 a 2010. Métodos: Realizou-se a análise retrospectiva dos prontuários de 25 pacientes em diálise peritoneal na referida clínica de diálise. Resultados: A população foi predominantemente masculina (76\%), de cor branca (80\%), com idade média de 65,88 anos. 0 diabetes mellitus foi a doença de base mais frequente $(36 \%)$, seguida de hipertensão arterial sistêmica (32\%), glomerulopatias (12\%), entre outras (20\%). Apenas um paciente iniciou a terapia renal substitutiva por diálise peritoneal. Em 92\% dos casos, o motivo de conversão para diálise peritoneal foi a exaustão de acesso vascular para hemodiálise. A média do tempo em diálise foi de 26,4 meses. A frequência geral de peritonite foi $88 \%$. Do total, três estavam em tratamento, dois (8\%) receberam alta, três (12\%) foram transferidos para hemodiálise e 17 (68\%) evoluíram a óbito. Conclusão: A minoria dos pacientes não apresentou peritonite. 0 modo inadequado da escolha de pacientes para diálise peritoneal e a falha na realização da técnica estão associados a maiores chances de ocorrência de peritonite. 0 estudo permitiu caracterizar uma população em diálise peritoneal ambulatorial contínua, possibilitando melhor planejamento do cuidado para evitar infecções.
\end{abstract}

Palavras-chave: epidemiologia; peritonite; diálise renal.

\section{Abstract}

Introduction: Peritoneal dialysis is a safe and effective alternative treatment for patients with terminal chronic renal failure. Since the introduction of peritoneal dialysis, peritonitis has been the main complication of this technique. Objective: To analyze the frequency of peritonitis in a hemodialysis center, located in the countryside of Paraíba, in Brazil, from 2005 to 2010. Methods: A retrospective analysis of medical records from 25 peritoneal dialysis patients in the clinic for dialysis was carried out. Results: The population was predominantly male (76\%), white (80\%), with a mean age of 65.88 years-old. Diabetes mellitus was the most frequent basis disease (36\%), followed by systemic arterial hypertension (32\%), glomerulopathies (12\%), among others (20\%). Only one patient started on renal replacement therapy for peritoneal dialysis. In $92 \%$ of the cases, the reason of conversion for peritoneal dialysis was exhaustion of vascular access for hemodialysis. The average time on dialysis was 26.4 months. The general frequency of peritonitis was $88 \%$. Of all, three patients were under treatment, two (8\%) were discharged, three (12\%) were transferred to hemodialysis, and $17(68 \%)$ died. Conclusion: The minor part of the patients did not present peritonitis during the period. The inappropriate way of patients' choice for dialysis peritoneal and the flaw in the accomplishment of the technique are associated with larger chances of peritonitis occurrence. The study characterized a population in continuous ambulatory peritoneal dialysis, enabling better planning of care to avoid infections.

Keywords: epidemiology; peritonitis; renal dialysis.

Recebido em: 20/07/2011

Revisado em: 12/09/2011

Aprovado em: 26/10/2011

Trabalho realizado em um Centro de Diálise no município de Sousa (PB), Brasil.

Universidade Estadual Vale do Acaraú (UVA) - Juazeiro do Norte (CE), Brasil.

2 Faculdade de Medicina de Cariri da Universidade Federal do Ceará (UFC) - Cariri (CE), Brasil.

3 Hospital Regional de Cajazeiras - Cajazeiras (PB), Brasil.

${ }^{4}$ Secretaria de Políticas Públicas para Mulheres - Cajazeiras (PB), Brasil.

Endereço para correspondência: Modesto Leite Rolim Neto - Avenida Tenente Raimundo Rocha, SN - Cidade Universitária - CEP: $63048-060$ - Juazeiro do Norte (CE), Brasil - E-mail: modestorolim@yahoo.com.br.

Fonte de Financiamento: nenhuma.

Conflito de interesse: nada a declarar. 


\section{Introdução}

A peritonite é a inflamação do revestimento da cavidade abdominal (peritoneu), provocada geralmente por uma infecção. O peritoneu, ou peritônio, é uma membrana fina e transparente que reveste os órgãos intra-abdominais e a face interna das paredes do abdômen. A peritonite é normalmente provocada por uma infecção propagada a partir de um órgão do abdômen. São causas frequentes as perfurações do estômago, do intestino, da vesícula biliar ou do apêndice. O peritoneu é bastante resistente à infecção. A menos que a contaminação seja muito persistente, não se desenvolve peritonite; o peritoneu tem tendência a se curar com o tratamento.

Em consequência de uma intervenção cirúrgica, pode-se desenvolver uma peritonite por várias razões. Uma lesão na vesícula biliar, no ureter, na bexiga ou no intestino, durante uma intervenção, também pode disseminar bactérias pelo abdômen. Nas operações em que se ligam dois segmentos intestinais, pode-se escapar conteúdo intestinal pelos pontos de sutura.

A diálise peritoneal (DP) favorece com frequência o desenvolvimento da peritonite. A causa habitual é uma infecção que abre passagem por meio dos drenos colocados no abdômen. A DP é uma forma de diálise intracorpórea, que aproveita a membrana peritoneal que reveste a cavidade abdominal, para realizar a função de filtração equivalente ao rim".

A DP é realizada mediante a infusão, por gravidade, de uma solução de diálise no espaço peritoneal, por meio de um cateter rígido ou flexível - cateter de Tenckhoff. Esta solução permanece na cavidade por tempo definido, no qual depois é drenada, também por gravidade, liberando as excretas metabólicas e o excesso de águal.

Portanto, pode ser feita pelo próprio paciente ou parente, em domicílio, após treinamento adequado. Na DP ambulatorial contínua (CAPD), as trocas das soluções devem ser realizadas com infusão de 2 a 2,5 L da solução dialisadora, obedecendo a um intervalo máximo de seis horas, sete dias da semana ${ }^{2}$. A DP intermitente segue o mesmo princípio da CAPD, porém, com duração de 12 horas, três vezes por semana ou 24 horas, duas vezes por semana, com intervalo entre as trocas de 30 minutos em ciclos de uma hora. A DP cíclica contínua é realizada no período noturno, utilizando uma máquina cicladora para efetuar as trocas das soluções automaticamente. Este método oferece vantagens sobre os demais, pois permite ao paciente um maior conforto e a realização de atividades diárias ${ }^{3}$. As principais complicações associadas à DP são a infecção do cateter e a peritonite ${ }^{4,5}$.

Um dos primeiros sinais de peritonite é a mudança da cor e aspecto do líquido drenado, que deve ser claro e fluido, com a peritonite, esse torna-se turvo e espesso. Outras manifestações como febre, dor abdominal, mal-estar, enjoos, vômitos e diminuição do apetite podem caracterizar a peritonite 6 .

Uma vez realizado o diagnóstico de peritonite, que é procedido por meio da observação dos sinais, sintomas e cultura do líquido dre- nado, inicia-se o tratamento com a administração via intraperitoneal de antimicrobianos, como a cefazolina ou cefalotina $(1 \mathrm{~g})$ associada com a ceftazidima ( $1 \mathrm{~g})$ e heparina $(1.000 \mathrm{U} / \mathrm{L})$, se houver presença de fibrina na solução drenada? .

A infecção do peritônio pode ocorrer devido à realização da técnica incorreta, o que permite que bactérias tenham acesso ao peritônio por meio do cateter (via intraluminal). Outra causa seria a penetração de bactérias presentes na pele no sítio de implantação do cateter (via periluminal), além de poder ser originada por via transmural, hematogênica e transvaginal, embora sendo menos frequente ${ }^{8}$.

Diversas medidas devem ser adotadas para prevenir ou minimizar as complicações associadas ao uso incorreto da técnica, com o intuito de melhorar a qualidade de vida e sobrevida do paciente em DP. O treinamento é um dos fatores cruciais para o sucesso da terapia, sendo fundamental na prevenção da peritonite. Essa tem desafiado os resultados clínicos e a manutenção dos pacientes em DP, que, apesar da redução de sua incidência, continua sendo a principal causa de fracasso da técnica?

Assim, o objetivo foi analisar a frequência de peritonite num centro de hemodiálise, situado no sertão da Paraíba, no Brasil.

\section{Métodos}

Trata-se de um estudo de caráter epidemiológico, em série de casos, descritivo e retrospectivo, de natureza quantitativa ${ }^{10,11}$, envolvendo prontuários de pacientes cadastrados no programa de CAPD em um Serviço de Nefrologia do sertão paraibano, de 2005 a 2010.

A investigação envolveu portadores de insuficiência renal crônica (IRC) de ambos os sexos, de diferentes classes sociais e raças, que realizaram DP como medida terapêutica para o restabelecimento da homeostasia. A amostra foi constituída de 25 pacientes submetidos à DP. Houve três pacientes que foram excluídos por mudança para a modalidade de hemodiálise, óbito ou alta.

A coleta de dados foi feita por meio da consulta aos prontuários dos pacientes atendidos por essa clínica entre os meses de setembro e dezembro de 2010. Nestes, as seguintes variáveis foram sublinhadas: sexo, idade, cor, tratamento inicial, tempo de permanência, destino dos pacientes (alta, transferência e óbito), comorbidades e complicações associadas ao tratamento.

Os dados foram tabulados e analisados utilizando o software EPIINFO, versão 3.4.3, sendo estes apresentados em tabelas.

O projeto de pesquisa foi aprovado pelo Comitê de Ética e Pesquisa da Universidade Federal do Ceará (UFC).

\section{Resultados}

No período de 2005 a 2010, o Serviço de Nefrologia teve 25 pacientes submetidos à DP. No momento da realização da coleta, três estavam em tratamento por CAPD e 22 já haviam saído do programa, por mudança para a modalidade de hemodiálise, óbito ou alta. 
Realizou-se a caracterização sociodemográfica dos 25 pacientes (Tabela 1). No que se refere à variável sexo, a população predominante foi masculina (76\%). No que tange a idade, a mediana foi de 65,88 anos e $64 \%$ tinham mais de 65 anos, portanto a população era idosa, mas $32 \%$ dos pacientes estavam na faixa etária dos 35 a 65 anos compondo o grupo dos economicamente ativos. Quanto à cor, predominou-se a branca $(80 \%)$.

Os pacientes avaliados residem com o familiar, auxiliando-os a desenvolverem as medidas de autocuidado, desde o diagnóstico da doença renal até o tratamento. A situação socioeconômica no binômio paciente-família merece destaque, pois o baixo poder aquisitivo favorece o aparecimento de complicações durante a terapêutica por CAPD (Tabela 2).

Quanto às comorbidades, a diabetes mellitus (DM) e a hipertensão arterial sistêmica (HAS) representaram as principais causas associadas à doença renal crônica, estando presente em $68 \%$ dos pacientes. De acordo com os dados, $36 \%$ dos pacientes apresentavam DM. A HAS constitui-se em um dos maiores problemas de saúde pública do Brasil e de outros países, encontrando-se entre as morbidades mais comuns do adulto e apresentando-se como uma das principais causas para a doença renal crônica.

A Tabela 3 mostra que, dos 25 pacientes avaliados, três (12\%) estão em tratamento, três (12\%) foram transferidos, dois (8\%) receberam alta e 17 (68\%) evoluíram ao óbito. Apenas um (4\%) iniciou terapia renal substitutiva (TRS) por meio de DP; os 24 pacientes restantes (96\%) iniciaram TRS por meio de hemodiálise e, posteriormente, foram convertidos para DP. Desta forma, os achados demonstram que a maioria dos pacientes tem como primeira opção a hemodiálise e demora a ingressar no CAPD, e só a praticam quando há necessidade de outra modalidade de tratamento. Isto pode ocorrer devido, na maioria dos casos, à exaustão de acesso vascular ou intolerância ao método hemodialítico, ou seja, iniciaram a DP somente quando tiveram problemas. Quanto ao tempo de permanência no programa de CAPD, observou-se que $28 \%$ dos pacientes permaneceram por um período de dois a cinco anos, seguido de $36 \%$ a menos de um ano de permanência no programa.

Constatou-se, ainda, que 12\% realizaram esta terapia há mais de cinco anos e $24 \%$ dos sujeitos praticam a modalidade CAPD há mais de um ano. Os pacientes estavam em DP há uma mediana de 26,4 meses e todos faziam CAPD. Neste período, $88 \%$ dos pacientes já haviam apresentado ao menos um episódio de peritonite. A frequência geral de peritonite foi de $88 \%$. A razão de conversão de hemodiálise para DP foi a exaustão de acesso vascular na maioria dos pacientes (92\%).

\section{Discussão}

A peritonite é ainda a grande responsável pela maioria dos óbitos nos pacientes em $\mathrm{DP}^{6}$. Esse fato pode ser representado por uma possível falha na realização do procedimento por parte dos profissionais ou cuidadores. Apesar disso, com a evolução do tratamento e a mudança
Tabela 1: Distribuição dos pacientes em tratamento por diálise peritoneal ambulatorial contínua, segundo características sociodemográficas. Sousa, Paraíba, 2010

\begin{tabular}{lc}
\hline Variáveis & $\mathrm{n}(\%)$ \\
\hline Sexo & $19(76)$ \\
Masculino & $6(24)$ \\
Feminino & \\
Idade & $1(4)$ \\
Até 35 anos & $8(32)$ \\
$35-65$ anos & $16(64)$ \\
Acima de 65 anos & \\
Cor da pele & $20(80)$ \\
Branca & $1(4)$ \\
Negra & $4(16)$ \\
\hline Parda
\end{tabular}

Tabela 2: Distribuição dos fatores de risco para doença renal crônica e doenças de base dos pacientes em tratamento por diálise peritoneal ambulatorial contínua. Sousa, Paraíba, 2010

\begin{tabular}{ll}
\hline Variáveis & $\mathrm{n}(\%)$ \\
\hline HAS & $8(32)$ \\
Diabetes mellitus & $9(36)$ \\
GNC & $3(12)$ \\
Cardiopatias & $2(8)$ \\
\hline Nefrolitíase & $1(4)$ \\
Lúpus eritematoso sistêmico & $1(4)$ \\
Rim policístico & $1(4)$ \\
Total & $25(100 \%)$ \\
\hline
\end{tabular}

GNC: glomerulonefrite crônica.

Tabela 3: Distribuição dos pacientes segundo destino, tratamento inicial, tempo de permanência no programa de diálise peritoneal contínua e ocorrência de peritonite. Sousa, Paraíba, 2010

\begin{tabular}{|cc|}
\hline Variáveis & $\mathrm{n}(\%)$ \\
\hline Destino & $3(12)$ \\
\hline Em tratamento & $3(12)$ \\
\hline Transferência & $2(8)$ \\
\hline Alta & $17(68)$ \\
\hline Óbito & \\
\hline Tratamento inicial & $24(96)$ \\
\hline Hemodiálise & $1(4)$ \\
\hline CAPD & \\
\hline Tempo para CAPD & $9(36)$ \\
\hline Menos de 12 meses & $6(24)$ \\
\hline 1 a 2 anos & $7(28)$ \\
\hline 2 a 5 anos & $3(12)$ \\
\hline+ de 5 anos & \\
\hline Peritonite & $22(88)$ \\
\hline Sim & $3(12)$ \\
\hline Não & \\
\hline
\end{tabular}


na conduta médica, esses pacientes estão sendo beneficiados com a administração de antibióticos durante as trocas de bolsas em seus domicílios, evitando longa permanência no ambiente hospitalar.

A DP pode ser utilizada como método de escolha inicial nas urgências dialíticas, mas muitas vezes não há disponibilidade de profissionais habilitados a realizar o implante do cateter de Tenckhoff em tempo hábil, nestas condições, a rapidez e a facilidade do implante do cateter duplo lúmen (CDL) para hemodiálise acabam prevalecendo ${ }^{1}$. No entanto, a maioria dos pacientes só foi colocada em DP como última opção, quando não havia mais acesso vascular para hemodiálise. Especificamente, apenas um dos 25 pacientes estudados iniciou TRS por meio de DP. A demora para confecção de fístula arteriovenosa também contribui para que estes pacientes permaneçam em hemodiálise por longos períodos. Nesta situação, o presente estudo mostrou que a maioria dos pacientes que iniciaram TSR por hemodiálise e depois foram convertidos para CAPD têm mais chances de apresentar ao menos um episódio de peritonite do que os demais. Devido ao caráter retrospectivo do trabalho, não foi possível encontrar registros detalhados sobre os agentes etiológicos das peritonites.

Quando a escolha de TRS é a DP, o treinamento para a realização da técnica torna-se um importante elemento de aproximação entre o paciente e familiar com a equipe do centro de diálise. Neste serviço, o treinamento é realizado em uma sala reservada dentro da unidade e sugere que seja de responsabilidade do enfermeiro. Provavelmente, a comunicação e a habilidade para incentivar o autocuidado de pacientes tenham destacado o profissional enfermeiro para realizar a seleção e o treinamento em $\mathrm{DP}^{12}$.

Como consequência do avanço técnico-científico e do uso de equipamentos modernos para o tratamento da IRC, amplia-se a competência dos enfermeiros em supervisionarem, coordenarem, interpretarem, decidirem e avaliarem as atividades realizadas em seu ambiente de trabalho. Entretanto, é necessário que os mesmos tenham, além da fundamentação científica e técnica, o conhecimento dos aspectos que levam em consideração a necessidade de aprendizagem por parte dos cuidadores e do próprio paciente para a realização do autocuidado e para obtenção do sucesso do tratamento ${ }^{9,12}$.
Constatou-se que, de 2005 a 2010, a ocorrência de um alto índice dos casos de peritonite, determinando o aumento do número de óbitos ${ }^{9,12-13}$. Com isto, reforça-se o planejamento da assistência em saúde, reconhecendo-se as características da população estudada, particularmente no que refere-se em obter um diagnóstico da realidade circundada e na redução das complicações da doença renal crônica e do tratamento por CAPD.

Considerando que diversos profissionais e familiares estão envolvidos no processo de DP desde a implantação do cateter até a manutenção do tratamento, todos devem ser orientados quanto à importância da qualidade da assistência para evitar infecções.

$\mathrm{O}$ tratamento da peritonite constitui uma urgência médico-cirúrgica, que exige sempre o internamento hospitalar do paciente. Por um lado, é necessário administrar antibióticos para combater a infecção e proceder a uma nova hidratação do paciente, mediante infusão intravenosa (gota a gota) para compensar a perda de líquidos por meio dos vômitos, bem como levar a cabo uma série de medidas destinadas a aliviar os seus sintomas: analgésicos para atenuar a dor, colocação de uma sonda nasogástrica para evacuar o conteúdo do tubo digestivo e aliviar a distensão abdominal, administração de oxigênio, possíveis transfusões de sangue etc. Por outro lado, pratica-se uma intervenção cirúrgica, indispensável no tratamento do distúrbio, a fim de solucionar a causa, eliminar o foco contaminante e drenar os líquidos infectados, procedendo até uma profusa lavagem do espaço peritoneal com soro fisiológico ${ }^{13}$.

Em suma, por tratar-se de uma população com alto risco de infecção, medidas devem ser tomadas para evitar a recidiva da peritonite. Para minimizar os casos de infecções em pacientes submetidos à DP, em especial a peritonite, e alcançar o sucesso do tratamento, alguns aspectos são indispensáveis, como acondicionamento adequado das bolsas, uso de máscaras, antissepsia rigorosa das mãos, ambiente apropriado e higienizado, manter portas e janelas fechadas, correta conexão dos equipos, infusão, permanência, drenagem e número de trocas de acordo com a prescrição e o desprezo do efluente em vaso sanitário ou ralo. Assim, esforços devem ser aplicados de maneira a estabelecer sistematicamente as medidas de prevenção e controle das complicações e contribuir significativamente para a qualidade de vida dos pacientes.

\section{Referências}

1. Blake PG, Daugirdas JT. Fisiologia da diálise peritoneal. In: Daugirdas JT, Blake PG, Ing TS. Manual de diálise. 3ª ed. Rio de Janeiro: Guanabara Koogan; 2003.

2. Ribeiro DF, Marques S, Kusumota L, Ribeiro RCHM. Processo de cuidar do idoso em diálise peritoneal ambulatorial continua no domicilio. Acta Paul Enferm. 2009;22(6):761-6.

3. Thomé FS, Barros E. Prevenção das doenças renais. In: Barros E, Manfro RC, Thomé, FS, Gonçalves LF. Nefrologia: rotinas, diagnóstico e tratamento. $2^{\mathrm{a}}$ ed. Porto Alegre: Artes Médicas; 1999.
4. Lobo JVD, Villar KR, Andrade Júnior MP, Bastos KA. Preditores de peritonite em pacientes em um programa de diálise peritoneal. J Bras Nefrol. 2010;32(2):156-64.

5. Kusumota L, Oliveira MP, Marques S. 0 idoso em diálise. Acta Paul Enferm. 2009;22: 546-50.

6. Abrahão SS, Ricas J, Andrade DF, Pompeu FC, Chamahum L, Araújo $\mathrm{TM}$, et al. Fatores de risco para peritonites e internações. J Bras Nefrol. 2010;32(1):100-6. 
7. Riella MC. Avaliação clínica e laboratorial da função renal. In: Riella MC. Princípios de nefrologia e distúrbios hidroeletrolíticos. $3^{\text {a }}$ ed. Rio de Janeiro: Guanabara Koogan; 1996.

8. Leehey DJ, Gandhi VC, Daugirdas JT. Peritonite e infecção do local de saída. In: Daugirdas JT, Blake PG, Ing TS. Manual de diálise. $3^{\mathrm{a}}$ ed. Rio de Janeiro: Guanabara Koogan; 2003.

9. Abreu RC, Pereira ERP, Gabriel DP, Caramori CA, Barreti P, Caramori JCT. Influência do treinamento na evolução da diálise peritoneal. J Bras Nefrol. 2008:30(2):126-31.

10. Medeiros JD. Redação cientifica: a prática de fichamentos, resumos, resenhas. $7^{a}$ ed. São Paulo: Atlas; 2005
11. Lakatos EM, Marconi MA. Metodologia do trabalho científico. $6^{a}$ ed. São Paulo: Atlas; 2006

12. Zilmer JGV, Schuartz E, Jardim VMR, Muniz RM, Buenos MEN, Feijó AM. Caracterização dos clientes em diálise peritoneal ambulatorial contínua CAPD da região sul do Brasil. Cogitare Enferm. 2009;14(2):318-23.

13. Rocha PN, Sallenave M, Casqueiro V, Campelo Neto B, Presídio S. Motivo de "escolha" de diálise peritoneal: exaustão de acesso vascular para hemodiálise? J Bras Nefrol. 2010;32(1):23-8. 